\title{
Recent Advances in Rheo-Optical Studies of Polymers in the Solid State
}

\author{
Richard S. STEIN \\ Polymer Research Institute, University of Massachusetts, \\ Amherst, Mass, U.S.A.
}

(Received August 20, 1984)

\begin{abstract}
Four recent developments for the study of the rheo-optical behavior of polymeric films are reviewed. These are (1) the use of the two-dimensional optical multichannel analyzer for the study of changes in superstructure by small angle light scattering, (2) the dynamic small angle Xray scattering technique for following time dependent morphology changes at a somewhat smaller level of size, (3) small angle neutron scattering for observing deformation at a molecular level, and (4) FTIR time resolved infrared spectroscopy which also permits molecular level observations. The use of these methods is illustrated with several examples involving both deformation and phase changes of amorphous and crystalline polymers.

KEY WORDS Rheo-Optics / Optical Multichannel Analyzer / Small-Angle Light Scattering / Small-Angle X-Ray Scattering / Small-Angle Neutron Scattering / Fourier Transform Spectroscopy / Dichroism / Dynamic / Time Resolved /
\end{abstract}

The objective of rheo-optical investigations is to employ optically related techniques to the study of rheological phenomena. The approach was inspired by the mentor of the author, the late Professor Arthur V. Tobolsky, who in about 1947, expressed the desire to "color a molecule red" in a solid polymer so that its changes could be observed as the polymer was deformed or otherwise changed. The objective is not easily met, but with the development of new and improved instrumental techniques, steady progress is being made.

The area of "rheo-optics" has been a fruitful field for collaboration between U.S. and Japanese workers. It was strongly emphasized in the first US-Japan Seminar in Polymer Physics held in Kyoto $^{1}$ in 1965 and several Japanese workers contributed to a special Symposium held in Amherst in $1963 .^{2}$ The laboratories of the University of Massachusetts and Kyoto University have been leaders in pioneering rheo-optical developments. A recent symposium at the Seattle meeting of the American Chemical Society in $1983^{3,4}$ included presentations of a series of papers by former students and associates of the author.
In this paper, four recent developments are discussed and illustrated, and a forecast is made concerning possible future trends in this field.

\section{PAST STUDIES}

\section{Light Scattering}

The dynamic birefringence technique was the first one to be developed. ${ }^{5,6}$ This was followed with dynamic light scattering ${ }^{7,8}$ and dynamic X-ray diffraction ${ }^{9,10}$ techniques. The early static light scattering studies on polymer films were carried out using a mercury lamp source ${ }^{11}$ and photographic recording. Exposure times of several hours were required to obtain patterns. Dynamic studies only became possible with the development of the laser, first a pulsed laser ${ }^{12}$ and then a continuous wave gas laser. ${ }^{13}$ High speed studies were accomplished through taking light scattering movies. ${ }^{14}$

The interpretation of light scattering patterns was accomplished using the theory for scattering from anisotropic spheres. ${ }^{15}$ This permitted the rapid measurement of spherulite size during the course of crystallization. ${ }^{16}$ The determination of the number, 
as well as the size of spherulites required the ability to theoretically account for disorder of crystal orientation within spherulites ${ }^{17,18}$ and for interspherulitic interference. ${ }^{19}$ The application of these theoretical advances was limited, however, by the difficulty of obtaining rapid quantitative time dependent intensity data, and only recently became feasible with the application of the optical multichannel analyzer (OMA) $)^{20,21}$ for intensity measurement.

The change in scattering patterns with orientation was first observed in Amherst ${ }^{15}$ and was subsequently applied extensively to the study of the orientation of polypropylene by Samuels. ${ }^{22,23} \mathrm{He}$ interpreted his results in terms of a semi-empirical approach, essentially involving an affine transformation of the theory ${ }^{15}$ for undeformed spherulites. A more correct theory, taking properly into account the reorientation of crystals within deformed spherulites was proposed in Amherst, first in two dimensions $^{24}$ and then in three. ${ }^{25}$ The theories involved parameters characterizing the relative contribution of various crystal reorientation processes accompanying sample deformation. This approach has been extensively developed in the laboratory of Kawai, ${ }^{26}$ and its application to the calculation of light scattering patterns has been continued by Matsuo. ${ }^{27}$ It is evident that the theories can rapidly become very complex, and involve so many parameters that their unique evaluation by light scattering alone becomes prohibitive. Thus, it becomes desirable to describe other features of the deformed polymer such as X-ray diffraction, birefringence and infrared dichroism in terms of the same models, and then to try to establish a set of model parameters which are consistent with the observations of all of these properties.

These theories are based upon the concept of affine deformation of spherulites. Such deformation would only occur if spherulites were mechanically isotropic. This is, of course, not so, because of the anisotropic arrangement of matter within spherulites. ${ }^{28}$ Crystalline lamellae lie radially so that amorphous and crystalline material interact effectively in parallel in this direction, whereas the modulus is a consequence of series addition in the tangential direction. Because of the lower amorphous modulus, the tangential modulus of the spherulite should be lower than the radial modulus. This should lead to a greater deformation in the equatorial regions of spherulites than in the radial part. A theory of this effect has been proposed by Wang, ${ }^{29}$ and attempts are in progress to apply these results to light scattering predictions. ${ }^{30}$

Another limitation of the deformation theories is that they are primarily concerned with the orientation of the crystalline component, whereas real polymer spherulites contain appreciable amorphous material which must make a significant contribution to the deformation process. No good understanding exists of the detailed relative orientation of the crystalline and amorphous regions within deforming spherulites. A first attempt in this direction is the Monte Carlo approach of Petraccone et al. ${ }^{31}$ The application of these concepts requires knowledge of structural parameters, such as numbers and lengths of tie chains and folds, for which values and experimental techniques are not readily available.

The variation of internal spherulite disorder with deformation has also not yet been described, and a theory for this is necessary to quantitatively account for the variation of the light scattering patterns during sample deformation. Extensive theoretical descriptions of light scattering from crystalline polymers have primarily been for spherulitic structures, whereas real systems often exhibit other morphologies. Preliminary theories of scattering from systems modelled as collections of anisotropic rods have been proposed in Amherst ${ }^{32}$ and developed in Kyoto. ${ }^{33}$

This discussion of the development of the light scattering technique provides a good illustration of the nature of the evolution of rheo-optical methods. The development is limited by the need for strong interaction between advances in theoretical approaches and experimental techniques. Thus, when a new experimental advance occurs a quantum advance in the method is possible. The four new techniques discussed in this article illustrate this principle.

\section{Dynamic Small-Angle X-Ray Scattering}

Small-angle X-ray scattering (SAXS) is traditionally used to characterize the structure of crystalline polymers. ${ }^{34}$ The scattering may be interpreted in terms of alternating crystalline and amorphous layers using the correlation function approach of Vonk ${ }^{35}$ or the paracrystalline method of Hosemann. ${ }^{36}$ For an unoriented polymer, the 
SAXS is cylindrically symmetrical about the incident beam, reflecting the fact that lamellar thickness and separation are independent of direction in such samples.

When a spherulitic polymer is stretched and the spherulites deform to become ellipsoids, the separation of the constituent lamellar changes in a manner reflecting the local deformation of the spherulite. The separation of those lamellae in the equatorial part of the spherulite which lie perpendicular to the stretch direction increases (Figure 1), while that for the lamellae in the polar part of the spherulite decreases. This leads to characteristic changes in the SAXS patterns, as studied, for example, by Hay and Keller. ${ }^{37}$ The results show that the idealized picture of Figure 1 is an oversimplification, ${ }^{38}$ and additional processes, such as buckling of lamellae in equatorial regions, must be introduced.

The quantitative rapid following of such changes requires rapid detection techniques. Film techniques are inconvenient, and point-by-point counting methods are not sufficiently rapid. A breakthrough has been the development of the position sensitive detector,${ }^{39}$ permitting the parallel accumulation of scattering intensities over a range of scattering angles. The initial one-dimensional detectors employed slit collimation and Kratkey optics. ${ }^{40}$ While this approach was satisfactory for isotropic samples, desmearing problems preclude their appli-
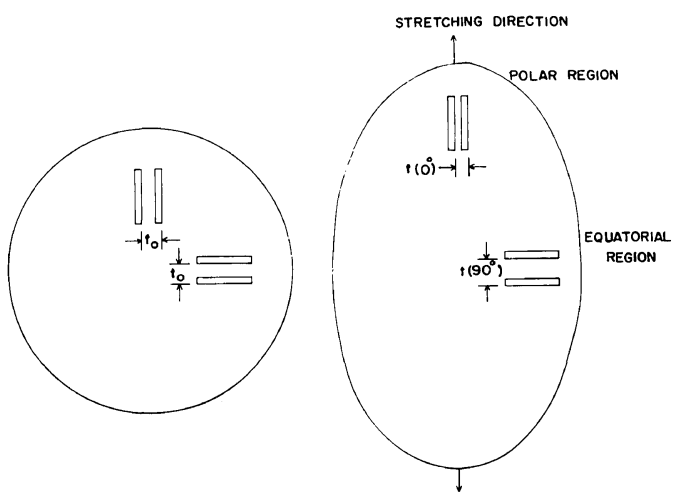

Figure 1. A model for the change in lamellar spacing occurring in deforming spherulites.

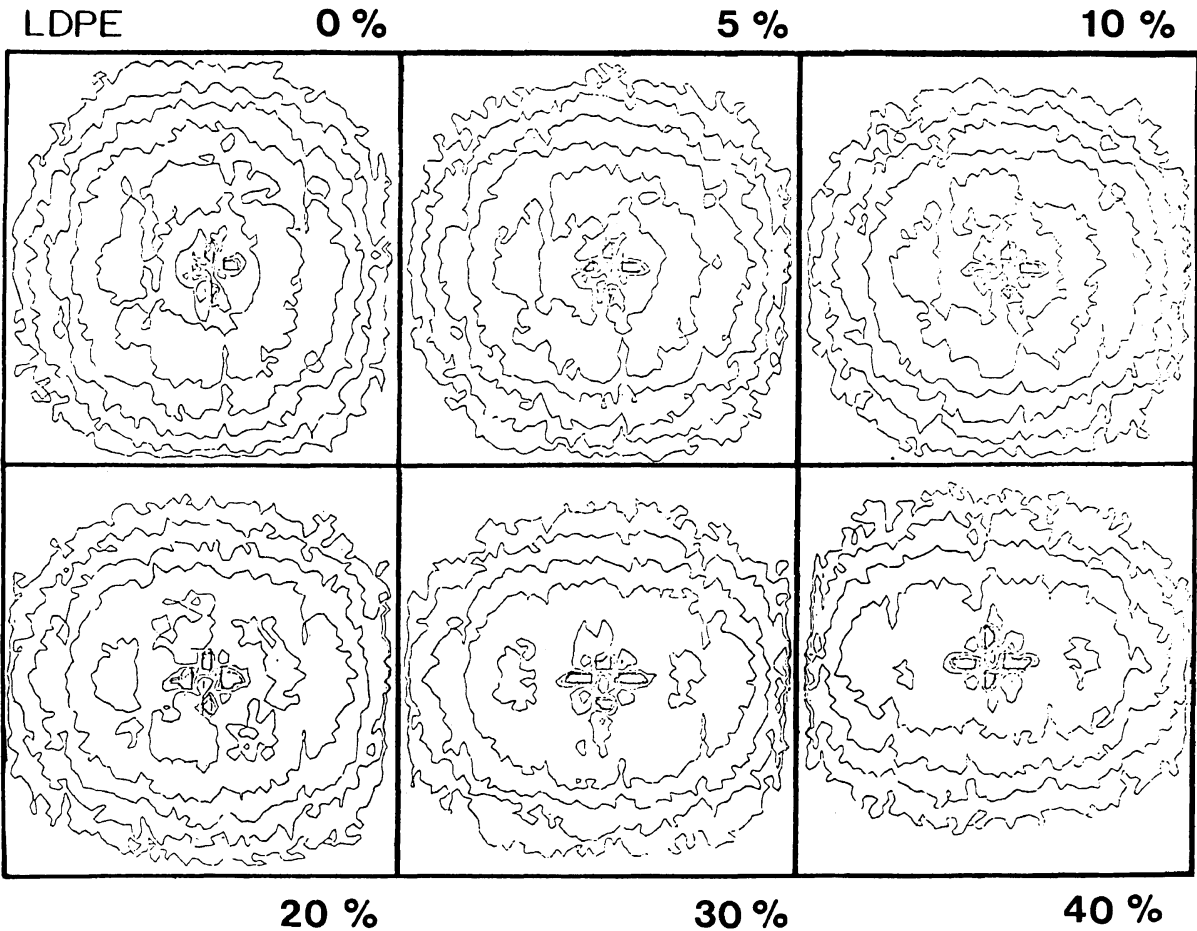

Figure 2. A series of SAXS intensity contours obtained using the Oak Ridge two-dimensional detector apparatus for polyethylene samples at different elongations. 
cation to partially oriented samples. Such samples require the use of pinhole collimation and, for rapid data aquisition, a two-dimensional detector.

An apparatus having this capability has become available at the National Center for Small-Angle Scattering Research at Oak Ridge National Laboratory (ORNL). ${ }^{41}$ A typical result showing SAXS intensity contours obtained during the deformation of a polyethylene sample is shown in Figure $2 .^{38}$ Preliminary interpretation of such data confirm the above-mentioned conclusion that internal deformation processes within spherulites are non-affine.

An objective is to utilize time-dependent SAXS measurements to complement the models employed to interpret the light scattering results. We shall describe how this may be done utilizing a signal averaging technique with the ORNL apparatus.

\section{Neutron Scattering Studies of Chain Conformation}

The small-angle neutron scattering (SANS) technique has assumed great importance in polymer physics, in that it permits the determination of chain orientation in the solid state. ${ }^{42}$ This is possible because of the ability to tag molecules by deuteration, which has a large effect on the scattering cross-section without appreciably affecting the thermodynamic or configurational properties. By this approach, one may resolve scattering contributions arising from inter- and intra-molecular interference, and thus determine single molecule properties in the condensed state in the presence of other molecules. It was initially thought that such studies required the labelled species to be dilute, but it was subsequently found that this restriction is not necessary, as demonstrated in a recent publication involving our group. ${ }^{43}$

It has been shown through studies on labelled crosslinked rubbers that the anisotropy of scattering may be determined through measurements on oriented systems. ${ }^{44}$ It is evident that such an approach may be applied to the study of chain orientation during the deformation of crystalline polymers, thus providing information about chain topology changes.

\section{Infrared Dichroism Measurements}

Starting with the early work of Fraser, ${ }^{45}$ the infrared dichroism method has been employed to characterize molecular orientation. Following earlier theoretica ${ }^{46}$ and experimenta ${ }^{47}$ efforts, Read and Stein $^{48}$ demonstrated the use of this technique for characterizing the orientation of crystalline and amorphous regions in polyethylene.

The IR dichroism technique is superior to the birefringence approach, in that it is specific. The birefringence arises from the overall molecular orientation, including molecules residing in both crystalline and amorphous regions. The separation of these contributions requires combining birefringence with another technique, such as X-ray diffraction, which characterizes crystal orientation. ${ }^{47}$ In this way, Amherst ${ }^{49}$ and Kyoto ${ }^{50}$ workers demonstrated differences in the dynamic orientation response of the crystalline and amorphous regions of polyethylene.

\section{RECENT DEVELOPMENTS}

\section{Solid State Light Scattering}

The availability of the OMA has greatly facilitated the rapid acquisition of quantitative light scattering data. A description of this device has recently been given in a thesis by Tabar, ${ }^{51}$ and applications have been described for studies of the isothermal crystallization of polyethylene terephthalate (PET) $)^{52}$ and the deformation of polyethylene (PE).$^{53}$ Some typical results of this study will be presented.

The spherulite size can readily be found from the angle, $\theta_{\mathrm{M}}$, at which the $H_{\mathrm{V}}$ (crossed polaroid) scattering maximum occurs at an azimuthal angle of $45^{\circ}$ using the equation first proposed by Stein and Rhodes ${ }^{15}$ for three dimensional spherulites

$$
\left[4 \pi\left(R_{\mathrm{S}} / \lambda\right) \sin \left(\theta_{\mathrm{M}} / 2\right)=4.08\right.
$$

The spherulite radius is $R_{\mathrm{S}}$, and $\lambda$ is the wavelength of the light (measured in the medium). The scattering angle $\theta_{\mathrm{M}}$ is also the value in the polymer medium and should be calculated from its value in air considering refraction at the polymer film-air interface.

The value of $\theta_{\mathrm{M}}$ can be determined by the computer interfaced to the OMA, so that the change in spherulite radius with time can be followed. Figure 3 shows a typical plot of the spherulite radius as a function of time during the isothermal crystallization of PET at $200^{\circ} \mathrm{C}$. It is seen that the value of $R_{\mathrm{S}}$ increases linearly with time but then asymptotically levels off toward a limiting value of $R_{\mathrm{SM}}$ at which the spherulites impinge as they become vol- 


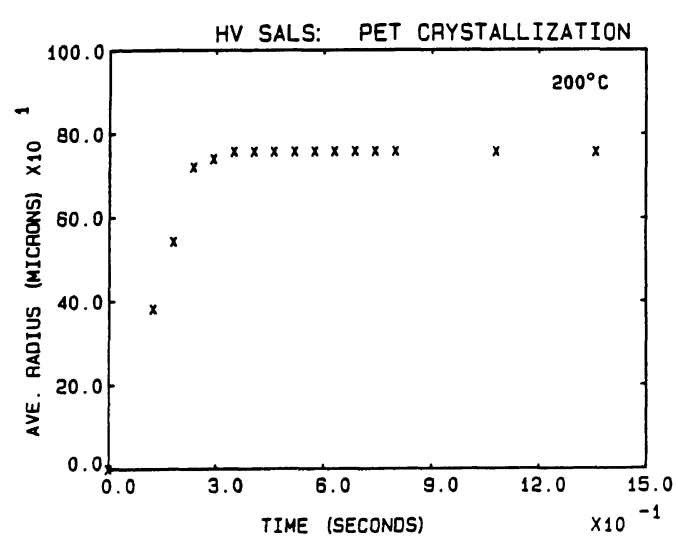

Figure 3. The variation of the spherulite radius, $R_{\mathrm{s}}$, with time during the isothermal crystallization of PET at $200^{\circ} \mathrm{C}$.

ume filling. If $N_{\mathrm{S}}$ is the number of spherulites per unit volume, then it follows that

$$
N_{\mathrm{S}}\left[(4 / 3) \pi R_{\mathrm{SM}}{ }^{3}\right] K=1
$$

The factor, $K$, takes into account that the spherulites are not complete spheres but are truncated because of impingement. Its value is dependent upon the statistics of nucleation, as has been discussed in recent papers. ${ }^{54,55}$

The intensity of scattering also increases with time during the course of crystallization. In addition to $R_{\mathrm{S}}$, this also depends upon $N_{\mathrm{S}}$ and upon the spherulite anisotropy $\left(\alpha_{\mathrm{R}}-\alpha_{\mathrm{T}}\right)_{\mathrm{S}}$ where $\alpha_{\mathrm{R}}$ and $\alpha_{\mathrm{T}}$ are the radial and tangential polarizabilities of the spherulite according to the approximate equation ${ }^{15}$

$$
\begin{gathered}
I_{\mathrm{HV}}(\theta)=K^{\prime} N_{\mathrm{S}} R_{\mathrm{S}}{ }^{6} \\
\left(\alpha_{\mathrm{R}}-\alpha_{\mathrm{T}}\right)^{2} F_{\mathrm{HV}}(U) G
\end{gathered}
$$

Here $F_{\mathrm{HV}}(U)$ is a scattering function for $H_{\mathrm{v}}$ polarization given by

$$
F_{\mathrm{HV}}(U)=\left(3 / U^{3}\right)(\sin U-U \cos U)
$$

The parameter $U=q R_{\mathrm{S}}$, where $q=(4 \pi / \lambda) \sin \left(\theta_{\mathrm{S}} / 2\right)$. The quantity $G$ is a disorder parameter which corrects for the fact that optic axes are not perfectly aligned within the spherulite. Its value has been discussed in the theory of Yoon and Stein. ${ }^{18}$ The anisotropy of the spherulite is given by

$$
\left(\alpha_{\mathrm{R}}-\alpha_{\mathrm{T}}\right)=\left(\alpha_{\mathrm{R}}-\alpha_{\mathrm{T}}\right)_{0} \cdot v_{\mathrm{CS}} f_{\mathrm{CS}}
$$

where $\left(\alpha_{R}-\alpha_{T}\right)_{0}$ is the intrinsic anisotropy of the polymer crystal, $v_{\mathrm{CS}}$ is the fraction crystallinity of

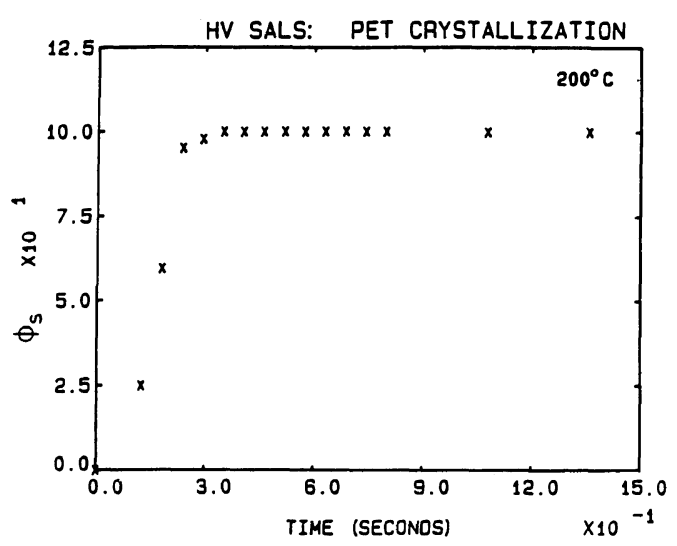

Figure 4. The variation of the degree of crystallinity of PET spherulites during its isothermal crystallization at $200^{\circ} \mathrm{C}$.

$H_{V}$ SALS: LDPE
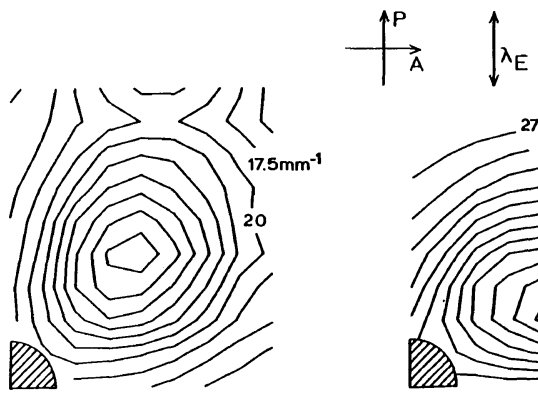

$$
\lambda_{E}=1.0 \quad \stackrel{\vdash-1}{0.25 \mu \mathrm{m}^{-1}} \quad \lambda_{E}=1.3
$$

Figure 5. $H_{\mathrm{v}}$ scattering intensity contours: (a) for an undeformed polyethylene sample; (b) for a sample stretched $130 \%$.

the polymer spherulite, and $f_{\mathrm{CS}}$ is an orientation function describing the degree of orientation of the polymer crystals with respect to the spherulite radius. In this approximation, the contribution to the spherulite polarizabilities arising from oriented amorphous material within spherulites of an unoriented sample is neglected.

From analysis of data according to these equations, it is possible to determine $N_{\mathrm{S}}, v_{\mathrm{CS}}$, and $G$ during the course of the crystallization. For example, Figure 4 shows the variation of $v_{\mathrm{CS}}$ with time during the course of the PET crystallization.

To illustrate the use of the OMA to follow sample deformation, one may see in Figure 5 the (sector averaged) OMA $H_{\mathrm{V}}$ pattern for (a) an undeformed PE sample, and (b) a sample deformed $50 \%$. It is 
seen that (1) the position of the maximum azimuthal intensity shifts from $45^{\circ}$ to larger azimuthal angles, and (b) the intensity at the maximum changes. The changes appear to be characteristic of the particular kind of sample, and differ, for example, for PE and polypropylene. Patterns have also been obtained for $V_{\mathrm{v}}$ polarization (parallel polarizers parallel to the stretching plane) and $H_{\mathrm{H}}$ polarization (parallel polarizers perpendicular to the stretching plane). One must then try to fit the scattering patterns for all three modes of polarization to the theoretically predicted patterns, as discussed above. This involves optimizing values of the parameters characterizing the internal crystal reorientation processes of tilting and twisting. A computer program was devised for finding values of the parameters which gave the minimum mean-squared difference between the theoretical prediction and experimental measurement in all three cases. It was found that even with optimum values of parameters, the meansquared error was rather large, indicating that the theory was inadequate to account for the experimets.

Consideration has been given as to how the theory might be improved. A prime difficiency is believed to be the affine assumption, and improvements have been made using the Wang theory as discussed above. Aslo, improvements are forthcoming as a result of incorporating the contribution arising from amorphous deformation within the spherulite and of changes in internal disorder. These improvements are facilitated through obtaining additional information about the lamellae and the amorphous chains by applying techniques like SAXS, SANS, and IR dichroism.

One might ask where future progress in SALS studies of polymer phase transformation may occur. The above studies, while fast compared with previous studies, are still relatively slow, taking a second or two for a measurement. The availability of scattered light intensity is not limiting. A very small laser has been employed for measurement, so the potential exists for substantially increasing light flux, if necessary. Speed of data acquisition is more limited by computer and detector electronics, and with some effort an appreciable decrease in acquisition time could be obtained. This would permit millisecond time scale experiments, in which case it may be worthwhile to revive the dynamic light scattering (DSALS) technique developed in
Amherst by Prud'homme and Hashimoto. ${ }^{7.8}$ In these studies, the SALS was observed during the sinusoidal deformation of PE samples. Point-bypoint scans of the variation of dynamic intensity (using phase-locked detection) were made as a function of azimuthal angle and $\theta$ using a photomultiplier photometer. This was a very tedious procedure, so extensive studies as a function of many sample variables were not feasible.

Another reason for not pursuing DSALS further at the time was the aforementioned limitation of theoretical interpretation. With the above indicated progress in the theoretical description of the spherulite deformation process, it appears again worthwhile to examine DSALS. It should prove useful in elucidating rheological mechanisms for systems having superstructure to accompany dynamic mechanical studies with DSALS. This becomes even more worthwhile in consideration of the development of other new rheo-optical techniques which may be applied in parallel.

If the DSALS technique is successful for spherulitic samples, it will then become interesting to extend it to other systems possessing superstructure. An example of interest in our laboratory is the study of thermotropic liquid crystalline polymers in mechanical, electrical or magnetic fields.

It will also be interesting to study phase change kinetics for non-spherulitic systems. Two examples under investigation in Amherst are the crystallization of stretched, crosslinked PE and the crystallization in a strong electric field of molten polyvinylidene fluoride $\left(\mathrm{PVF}_{2}\right)$.

\section{Progress in Rheo-Optical Applications of SAXS}

A servo-controlled deformation apparatus has been installed on the $10 \mathrm{~m}$ two-dimensional detector SAXS apparatus at ORNL. The deformation is programmed using the same computer which is employed for data acquisition. The counts are distributed to memory locations in a manner dependent upon sample strain, and the signal is accumulated over many cycles of periodic strain.. A given memory location receives the count at the same phase angle of strain for each cycle. In this way, the signal may be averaged. While the number of counts received in a single strain cycle is usually insufficient to give a statistically significant result, the accumulated signal over many cycles is sufficient. The best way to analyze the data appears to 
be to Fourier analyze the X-ray counts with respect to the strain. For sinusoidal strain the signal also varies sinusoidally, but generally with a phase angle with respect to the strain which is dependent upon the phase angle of the structural response which gives rise to the scattering.

As discussed earlier, changes in the SAXS from crystalline polymers may arise from (1) changes in orientation of lamellae, (2) changes in lamellar thickness or separations, (3) twisting of lamellae, and (4) changes in void content. The dynamic SAXS technique (DSAXS) is capable of resolving the dynamics of these separate processes.

The total amount of scattering from a material is conserved if only changes in orientation or location occur. For an isotropic material, this can be expressed in terms of the integrated intensity or invariant, $Q$, defined as ${ }^{34}$

$$
Q=\int_{0}^{\infty} I(q) q^{2} \mathrm{~d} q
$$

For a two-phase system containing volume fractions $v_{\mathrm{A}}$ and $v_{\mathrm{B}}$ of phases $\mathrm{A}$ and $\mathrm{B}$ with sharp boundaries, and having electron densities $d_{\mathrm{A}}$ and $d_{\mathrm{B}}$, the invariant is given by

$$
Q=v_{\mathrm{A}} v_{\mathrm{B}}\left(d_{\mathrm{A}}-d_{\mathrm{B}}\right)^{2}
$$

For an oreinted system, one must replace the onedimensional integral of eq 6 by a three-dimensional integral over all of reciprocal space.

For DSAXS experiments, a dynamic invariant, $Q_{\mathrm{D}}$, may be determined through integration of the time detendent intensities over reciprocal space. If the amount of scattering material remains constant in the deformation experiment, $Q_{\mathrm{D}}$ should not change. This will not be the case if, for example, voids open and close during the deformation. For a two-phase system containng a volume fraction $v_{\mathrm{v}}$ of voids, eq 7 may be generalized to give

$$
Q=v_{\mathrm{A}} v_{\mathrm{B}}\left(d_{\mathrm{A}}-d_{\mathrm{B}}\right)^{2}+v_{\mathrm{A}} v_{\mathrm{V}} d_{\mathrm{A}}^{2}+v_{\mathrm{B}} v_{\mathrm{V}} d_{\mathrm{B}}^{2}
$$

(Note that $d_{\mathrm{V}}=0$ ). Thus, if $v_{\mathrm{V}}$ changes with deformation, $Q$ will change.

Figure 6 shows some results of DSAXS experiments on a high density PE sample. ${ }^{56}$ The intensity change is represented by a complex number $\Delta I^{*}$ which is resolved into real and imaginary parts, $\Delta I^{\prime}$ and $\Delta I^{\prime \prime}$ according to

$$
\Delta I^{*}=\Delta I^{\prime}+i \Delta I^{\prime \prime}
$$

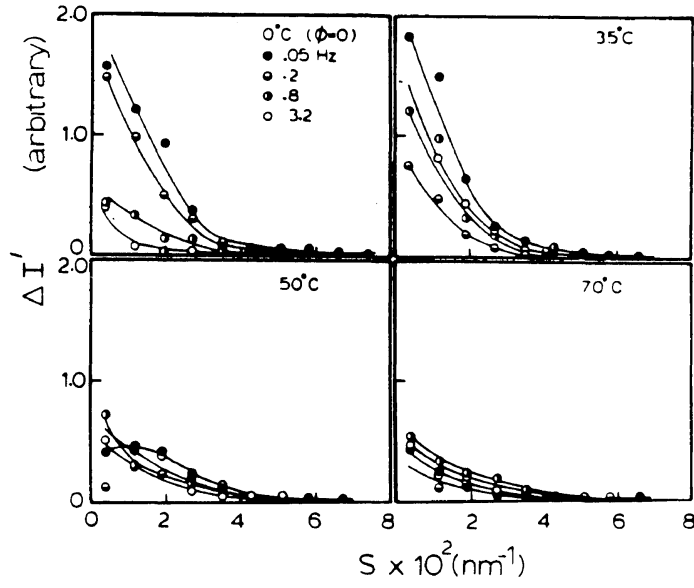

Figure 6. Dynamic SAXS for a sample of high density polyethylene at a series of frequencies and temperatures.

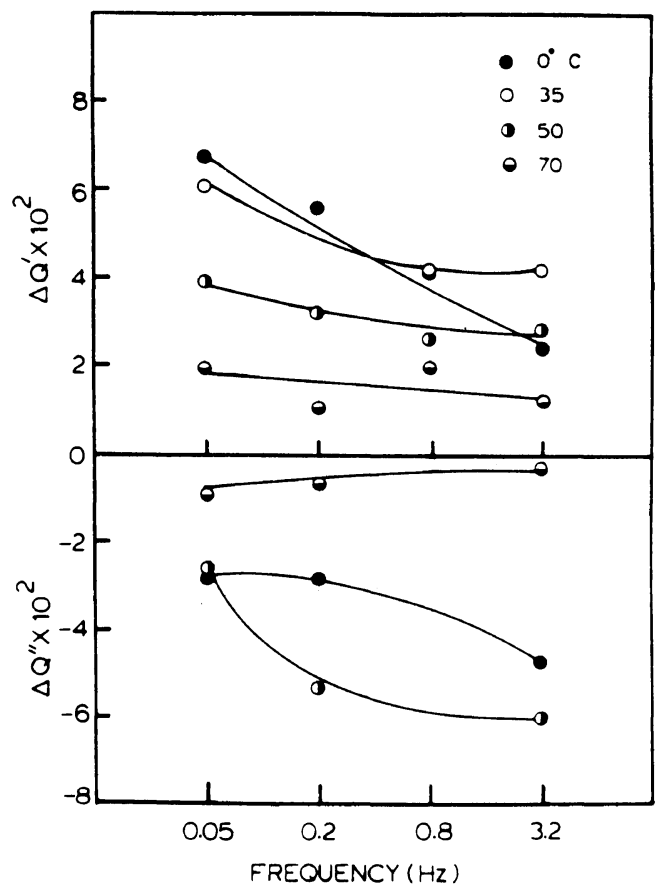

Figure 7. The real and imaginary part of the dynamic invariant.

These represent the parts of the intensity change which are in and out of phase with the strain, so that the phase angle, $\gamma$, is given by

$$
\tan \gamma=\left[\left(I^{\prime \prime}\right) /\left(I^{\prime}\right)\right]
$$


It is evident that the dynamic response is frequency dependent, indicating a time dependent structural response. A dynamic invariant was calculated from this data, and is plotted in Figure 7. It is evident that its value is finite and dependent upon frequency and temperature. We believe that this response is primarily due to dynamic void formation. The contribution decreases at higher temperature, and is suppressed by introducing a solvent believed to fill the voids.

By making measurements at elevated temperatures or on low density PE samples, the void contribution is reduced in importance and one may obtain meaningful dynamic data on lamellar changes. Such studies are in progress. In the future, it is expected that the DSAXS technique will be greatly facilitated by the availability of synchrotron X-ray sources. These are several orders of magnitude brighter than conventional sources and should correspondingly reduce data acquisition times. Similar advantages will ensue from the use of very high amperage rotating anode sources such as are becoming available in Japan. At high X-ray intensities, one becomes detector limited. Conventional gas position sensitive detectors are too slow to take advantages of the high pulse rates. Solid state detectors, such as reticon diode arrays coupled with fast electronics, are being developed which should greatly increase detection speeds.

\section{Studies of Rheology of Chain and Crystal Orien- tation by SANS}

For unoriented samples, the SANS intensity is cylindrically symmetrical about the incident beam and is independent of azimuthal angle. This is a consequence of polymer molecules being (on the average) spherically symmetrical for undeformed polymers. This may be seen from Figure 8a, which plots intensity contour lines for a polystyrene (PS) sample which contains $6 \%$ of deuterium labelled PS (PSD). It is seen that these contours are circular, verifying that molecular dimensions, which may be expressed in terms of the single molecule radius of gyration, $R_{\mathrm{G}}$, are independent of direction.

The value of $R_{\mathrm{G}}$ may be determined from the $q$ variation of scattering $[q=(4 \pi / \lambda) \sin (\theta / 2)]$ according to the Guinier ${ }^{34}$ or, better, the $\mathrm{Zimm}^{57}$ equation

$$
I(q)=I(0)\left[1-\left(R_{\mathrm{G}}^{2} / 3\right) q^{2}+\cdots\right]
$$


Figure 8. Intensity contour lines for SANS from a PS sample containing 6\% PSD: (a) an unoriented sample; (b) a sample drawn $4.2 \times$ by extrusion orientation.

where $I(0)$ is the value of $I(q)$ at $q=0$. Thus, the slope of a plot of $I(q) v s . q^{2}$ should be $\left(R_{\mathrm{G}}^{2} / 3\right)$ at small $q$. Often, one plots $I(q)^{-1}$ rather than $I(q)$ which, at small $q$, gives the same slope but with reversed sign. Such a plot is only linear at small $q$, so it is important to obtain data at sufficiently small $q$ (the Guinier region) that this be so. Data at small $q$ arises from intramolecular interferences between parts of molecules separated by large distances, and provides information about the "global" dimensions of the molecule. The neglected larger $q$ data arises from smaller distances and characterizes more local conformations as discussed later.

In Figure $8 b$, a contour is given ${ }^{58}$ for the same sample as in Figure 8a. The sample has been drawn $4.2 \times$ by the extrusion technique developed in Amherst by Porter ${ }^{59}$ It is seen that, in this case, the intensity contour lines are extended in a direction perpendicular to the stretching direction (vertical), 
indicating that the radius of gyration is directionally dependent. As is seen from eq 11, an $I(q)$ falling off more rapidly with $q$ corresponds to a larger $R_{\mathrm{G}}$. In fact, dimensions often become great enough in the $\|$ direction so that low $q$ data is lost behind the beam stop, and special procedures become necessary for extrapolating into this region. Thus, the data gives the expected result that $R_{\mathrm{G}}$ in the stretching direction $(\|)$, designated as $R_{\|}$, is greater than $R_{\perp}$ in the $\perp$ direction. A plot of the variation of $R_{\|}$and $R_{\perp}$ $v s$. extension ratio, $e$, for this sample is given in Figure 9. The lines on the plot are not fits to the experimental points but are theoretical lines calculated according to the affine model, where

$$
R=R_{\mathrm{G}}^{0} e
$$

and

$$
R=R_{\mathrm{G}}^{0} e^{-1 / 2}
$$

This model presumes that the molecular dimensions change in the same ratio as do the external dimensions of the sample. It is seen that it fits the data well, except for the highest extensions.

These results may be explained by considering the molecules in the melt as forming an "entanglement network" $" 60$ where the mean positions of the entanglements transform affinely (as with a crosslinked rubber) and for which the entanglements preserve their integrity during deformation. The deviation at high elongations may be understood by considering that entanglement slippage may occur at high strains.

An alternate model is in terms of the "reptation" concept $^{61-63}$ in which the molecules are thought to reside in a "tube" which threads through the entanglements of neighboring molecules. The tube is postulated to deform affinely when the polymer is deformed, and subsequent relaxation processes are interpreted in tersm of the one dimensional diffusion of the molecule in the tube, with subsequent tube disappearance and renewal.

It must be realized that the PS studies is not crosslinked, so that relaxation processes should occur following sample extension which ultimately lead to the value of $R_{\mathrm{G}}$ returning back to its initial value. The reason the affine prediction appears to work is that the relaxation time is sufficiently long so that the entanglement slippage process does not occur to a significant extent during the time of the experiment. The experiment was carried out by

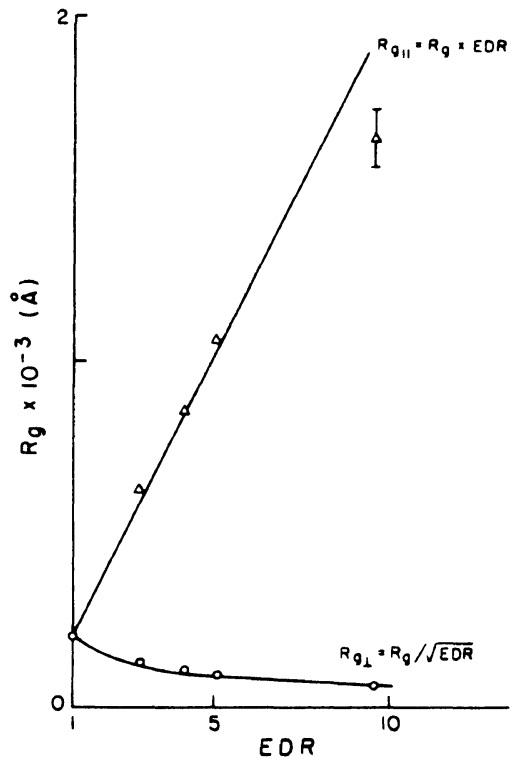

Figure 9. The variation of the parallel and perpendicular components of $R_{\mathrm{G}}, R_{\|}$and $R_{\perp}$ with sample extension ratio $e_{\mathrm{S}}$ for an extrusion oriented polystyrene sample.

cooling the extruded melt with an air blast as rapidly as possible as it emerged from the capillary of the extrusion apparatus. As soon as the temperature was lowered below the glass transition temperature, $T_{\mathrm{G}}$, of the polymer, relaxation processes were suppressed.

With lower molecular weight samples, relaxation is faster, so that it no longer becomes possible to prevent appreciable relaxation during the time required for quenching. Thus, while for the high molecular weight (about 500,000) sample corresponding to the data of Figure 9, the affine model works well, significant deviations are found for a lower molecular weight $(80,000)$ sample, where the molecular weight is not much greater than the mean molecular weight between entanglements. ${ }^{64}$

If the sample is heated above its $T_{\mathrm{G}}$, maintaining a constant sample length, relaxation will then commence, and $R_{\mathrm{G}}$ will return toward its unperturbed value. Such samples have then been quenched to below $T_{\mathrm{G}}$, freezing in the $R_{\mathrm{G}}$ corresponding to the state of relaxation, and SANS measurements were made, allowing the determination of $R_{\|}$and $R_{\perp}$ as a function of time. Similar studies have been conducted in France by F. Boue. ${ }^{65} \mathrm{~A}$ molecular exten- 
sion ratio, $e_{\mathrm{M}}(t)$ may be defined as

$$
e_{\mathrm{M}}(t)=R_{\mathrm{G}}(t) / R_{\mathrm{G}}(t=0)
$$

A question is whether the variation of $e_{\mathrm{M}}(t)$ with time corresponds to other measures of molecular relaxation.

A method for following molecular extension and relaxation extensively employed in both Amherst and Kyoto is the birefringence technique..$^{5,6.47 .66-69}$ The bireringence, $\Delta n$, is related to segmental orientation by

$$
\Delta n=\Delta n_{0} P_{2}
$$

where $\Delta n_{0}$ is the intrinsic birefringence that would be achieved if the segments were perfectly oriented, and $P_{2}(s)$ is the Hermans orientation function ${ }^{70}$ of the segment defined by

$$
P_{2}(s)=\left[3\left\langle\cos ^{2} \theta_{I}\right\rangle-1\right] / 2
$$

where $\theta_{I}$ is the angle between the $I$-th segment and the stretch direction. For a Gaussian chain in a crosslinked network at low extensions, $P_{2}(s)$ is given by $^{71}$

$$
P_{2}(s)=\left[1 /\left(5 n_{\mathrm{S}}\right)\right]\left[e_{\mathrm{E}}^{2}-\left(1 / e_{\mathrm{E}}\right)\right]
$$

where $n_{\mathrm{S}}$ is the number of (Kuhn) segments between crosslinking points, and $e_{\mathrm{E}}$ is the sample extension ratio $\left[e_{\mathrm{E}}=\left(L / L_{0}\right)\right]$ where $L$ and $L_{0}$ are the sample length and unstretched length, respectively. For unoriented samples, $\left\langle\cos ^{2} \theta_{I}\right\rangle=1 / 3, P_{2}(s)=0$, as is predicted by eq 16 when $e_{\mathrm{E}}=1$. For a perfectly oriented sample, $P_{2}(s)=1$. A birefringence measure of the state of orientation is defined as

$$
e_{\mathrm{B}}(t)=\left[\Delta n(t) /\left(\Delta n_{0}\right)\right]=P_{2}(s, t)
$$

It is evident that if the affine model with no slippage of crosslinks applies, so that $e_{\mathrm{M}}=e_{\mathrm{E}}$ in the $\|$ direction, a combination of eq 16 and 17 gives

$$
e_{\mathrm{B}}=\left[1 / 6\left(n_{\mathrm{S}}\right)\right]\left[e_{\mathrm{M}}^{2}-\left(1 / e_{\mathrm{M}}\right)\right]
$$

Thus, it is evident that $e_{\mathrm{M}} \neq e_{\mathrm{B}}$, so that this measure of orientation by birefringence is not expected to equal that by SANS.

Furthermore, eq 16 was derived using the assumption that the portion of the chain between two crosslinks could assume random conformations and is describable by Gaussian statistics. Depending upon the relaxation model, this is not necessarily so. For example, with the reptation model, deviations should occur as chains diffuse within the "tube."

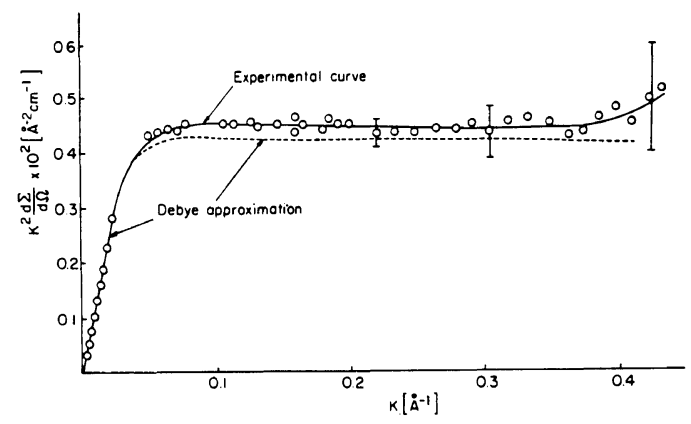

Figure 10. A typical Debye plot of $q^{2} P(q) v s . q$ for a $5 \%$ protopolystyrene in deuteropolystyrene (-- ) calculated from the Debye model for Gaussian coil. In the figure, ' $q$ ' is designated as ' $K$ '.

Such deviations lead to deviations from the Gaussian scattering prediction at higher values of $q$.

The scattering from a spherically symmetrical molecule may be calculated according to Debye ${ }^{72}$ from

$$
\begin{aligned}
P(q) & =[I(q) / I(0)] \\
& =\int_{0}^{\infty} p(r)[\sin (q r) /(q r)] r^{2} \mathrm{~d} r
\end{aligned}
$$

where $P(q)$ is called "the single molecule interference function" and $p(r)$ is the probability that two segments of a molecule will lie a distance $r$ apart. For a Gaussian coil, $p(r)$ is calculated from gaussian statistics according to

$$
p(r)=\left[B_{I J} / \pi\right]^{3 / 2} \exp \left[-B_{I J} r^{2}\right]
$$

where

$$
B_{I J}=3 /\left(2 r^{2}\right)=3 /\left(2 n_{I J} S^{2}\right)
$$

$r_{I J}^{2}$ is the mean-squared separation of the $I$-th and $J$ th sements which are separated along the chain by $n_{I J}$ Kuhn statistical segments, each of length $S$. The sum over $I$ and $J$ is over all pairs of segments constituting the chain. By substituting eq 20 into 19 , Debye obtained for the scattering function for a Gaussian chain

$$
P(q)=\left(2 / u^{2}\right)\left(e^{-u}-1+u\right)
$$

where $u=q^{2} \bar{R}^{2} / 6$ and $\bar{R}^{2}$ is the mean-squared endto-end length of the molecule. For an unbranched molecule, this is related to the radius of gyration by $R_{\mathrm{G}}^{2}=R^{2} / 6$, so $u=q^{2} R_{\mathrm{G}}^{2}$. It is seen that at large $q$ when $u$ is large, eq 22 reduces to 


$$
\operatorname{limit}[P(q)]=2 / u=2 /\left[q^{2} R_{\mathrm{G}}^{2}\right]
$$

Thus, a plot of $q^{2} P(q) v s . q^{2}$ should start at zero at $q=0$ and increase with increasing $q$, reaching a limiting value of $1 /\left(R_{\mathrm{G}}^{2}\right)$ at large $q$. This is called a "Debye plot" and serves as a test for the Gaussian chain model. A typical Debye plot is shown in Figure 10, where it is seen that an initial asymptotic limit is reached, but that deviations occur at larger $q$.

The reason for the deviations is that the large $q$ data is sensitive to interference between waves scattered from closely spaced segments. Equation 10 , based upon the Gaussian approximation is only valid provided that the number of segments, $n_{I J}$, connecting the interfering $I$-th and $J$-th segment is large. This will not be so for many of the segment pairs contributing to scattering at small $r$. In this case, their separation will not be adequately described by eq 10 . Such chain sections containing a small number of segments are not as flexible as a Gaussian coil and will be more rod-like in their statistics for which $P(q)$ varies with $1 / q$ at large $q$ rather than $1 / q^{2}$. Thus, a Debye plot of $q^{2} P(q)$ will not become asymptotic but will be proportional to $q$ at large $q$. The $q$ at which this occurs depends upon chain stiffness (as expressed in terms of the segment length, $S$ ) and is related to the reciprocal of the "persistence length" of the chain.

While the Gaussian description of a chain is independent of its chemical structure, and all Gaussian chains have the same form of $P(q)$, the small $r$ statistics of real chains depends upon the actual molecular geometry, bond lengths and angles, isomerism, etc. Equation 19 is still valid, but one must use a better $p(r)$ than eq 20 . Flory and coworkers have done many such calculations using the rotational isomer model. ${ }^{73,74}$ Flory et al. have shown that $P(q)$ significantly differs in the high $q$ region for syndiotactic and isotactic poly(methyl methacrylate) reflecting a local conformational difference.

Thus, it is seen that there are two ways of obtaining $R_{\mathrm{G}}$; (1) from Zimm or Guinier plots in the Guinier region at small $q$, and (2) from the assymptotic value of $q^{2} P(q)$ in the Debye region at large $q$. The first approach is sensitive to the large scale or global dimensions, while the second is dependent upon the small scale or local structure. For a truly Gaussian chain, the two approaches should yield the same $R_{\mathrm{G}}$.

For an oriented polymer, $\|$ and $\perp$ data could be analyzed by both approaches, indicating whether the molecular deformation is similar at both long and short differences. For relaxation studies one could follow molecular extension ratios $e_{\mathrm{M}}(L)$ or $e_{\mathrm{M}}(S)$ for long $(L)$ and short $(S)$ distances, defined as in eq $13 \mathrm{~b}$, but using $R_{\mathrm{G}}$ values obtained in the Guinier and Debye regions, respectively. If the chains remained Gaussian throughout the relaxation process, $e_{\mathrm{M}}(L)$ and $e_{\mathrm{M}}(S)$ should be identical and remain so throughout the relaxation. Preliminary indications are that short range order relaxes out faster than does long, so that $e_{\mathrm{M}}(L)$ decreases more slowly than does $e_{\mathrm{M}}(S)$.

The situation in a crystalline polymer is more complex. In this case, the SANS arises from portions of the chain traversing both crystalline and amorphous regions. The theory for measuring chain dimensions by SANS using solutions of deuterated chains in a hydrogenous matrix (or vice versa) requires that the two species be randomly mixed. Due to a $4^{\circ} \mathrm{C}$ crystalline melting point difference between the species in PE (PEH and PED), segregation does occur during crystallization, leading to an intermolecular interference contribution to the scattering and an erroneous $R_{\mathrm{G}}{ }^{75}{ }^{75}$ A test of segregation is to determine the molecular weight from the absolute value of $I(q)$ at $q=0$ and compare it with its value for the isolated molecule as determined by solution methods.

There is some indication that this aggregation is broken up upon stretching, ${ }^{76}$ so perhaps the problem is not so serious with oriented samples. A partial solution to the segregation problem is to employ partly deuterated rather than completely deuterated species, as is being done for PE by Graessley and Crist. ${ }^{77}$ There is indication that the problem is not so serious in polypropylene ${ }^{78}$ or in molecules with less hydrogen content than PE.

Studies with PE and other crystalline polymers have been largely directed toward the characterization of the crystal fold surface, and to establish the adjacency of chain fold re-entry. Monte Carlo calculations of scattering from such crystals considering re-entry statistics have gained considerable attention, and the studies by Yoon and Flory ${ }^{79}$ are noteworthy. Their comparison with experiments ${ }^{80}$ suggests that adjacent re-entry is relatively infrequent. 




Figure 11. The variation of $R_{\|}$and $R_{\perp}$ with sample extension ratio for an extrusion oriented sample of high density polyethylene.

We have attempted to characterize the orientation of PE through observation of the anisotropy of SANS upon stretching. Figure 11 shows the variation of $R_{\|}$and $R_{\perp}$ with sample extension for an extrusion oriented sample of HDPE containing some PED ${ }^{81}$ It is evident that the molecular extension falls considerably below the affine prediction. This is not surprising, since the orientation is a composite process involving contributions from both the crystalline and amorphous parts. We have also examined the high $q$ behavior and found that, while there is no theoretical reason to expect the Debye function to apply since we are not dealing with Gaussian coils, the values of $R_{\mathrm{G}}$ calculated from the assymptotic behavior of $q^{2} P(q)$ agree reasonably well with those obtained from the Guinier region. ${ }^{81}$

Preliminary attempts have been made to describe the $P(q)$ for a crystalline polymer for two cases: (1) at low elongations where crystalline and amorphous regions preserve their integrity during deformation, and no transfer of chains from crystalline to amorphous regions or recrystallization occurs; and (2) at higher elongations where recrystallization may occur and folded chain crystals transform into extended chain crystals. With case (2), at sufficiently high elongations, one approaches as the limit a completely chain extended oriented polymer.

For case (1), the chains in the crystalline and amorphous regions are treated separately. The only change occurring in the crystalline chain stems is that due to the change in crystal orientation and location. These processes may be characterized by
WAXD and SAXS. The amorphous chains are divided into four types: (a) tight loops, (b) loose loops, (c) tie chains, and (d) chain ends. The tight loops are constrained to move in a manner dependent upon the motion of the crystal with which they are associated. The loose loops and chain ends have a certain degree of conformational freedom and are able to assume a variety of conformations, subject to the restraint that they are anchored to the crystal at one or two points, and the rest of the chain may assume random conformations limited by the barriers of the surrounding crystal surfaces. These barriers will move during deformation since, for example, the distance between lamellar surfaces changes in a manner which may be characterized by SAXS. The tie chains bridge two crystals, so that their displacement vectors, connecting the points at which the chain is tied to the crystal, will change in direction and orientation as the intercrystalline spacing changes. Thus, their conformational entropy will change.

The calculation of these chain properties requires the specification of the number of chains of each type and their lengths. These values depend upon the morphology in the undeformed state since it is assumed that no changes in these quantities occur upon deformation. They are dependent upon the nature of the crystallization process and the molecular weight of the polymer. For example, high molecular weight samples lead to a higher probability that a chain will find itself participating in two or more crystals and add to the tie chain concentration. This will be more likely when crystallizing at high degrees of supercooling, when crystallization is rapid and chains have less time to "sort themselves out." With rapid crystallization, regular folding is less likely, so tight fold concentration is less.

It is also possible to deduce information about amorphous chain types from other physical measurements. For example, the amorphous orientation function, $\left(P_{2}\right)_{\mathrm{AM}}$, can be deduced from IR dichroism measurements or from a combination of birefringence with WAXD. As shown by Petraccone et al..$^{31}$ its value depends upon amorphous state parameters. A recent publication has appeared $^{82}$ suggesting a means for estimating the number of tie chains from SANS observations.

It is anticipated that with the coming on line of higher flux sources, the acquisition of SANS data 
will be facilitated. It can now take from minutes to hours to obtain an intensity contour for an oriented sample. This is tedious for following timedependent processes, and precludes, in most cases, real time studies. It may become feasible to carry out real time measurements for samples subjected to periodic strain using signal averaging techniques, much as described above for DSAXS. The development of pulsed sources will be helpful for such studies. It should be possible to synchronize the oscillation frequency of the sample strain with the pulse rate and vary the phase angle between the two.

Pulsed sources will also be useful for phase transformation studies. The interval between pulses may be great enough for time-of-flight sorting of the wavelengths of the scattered neutrons. This permits a scanning in $q$ space from measurements of scattering at a single angle.

\section{Recent Contributions to Rheo-Optics from Dynamic Infrared Dichroism}

As previously discussed, the infrared dichroism (IRD) technique is of great value in determining the orientation functions of specific portions of polymer molecules or systems. The absorbance is conventionally measured for radiation polarized parallel $[A(\|)]$ and perpendicular $[A(\perp)]$ to the stretching direction, and the dichroism, $D$, is defined as

$$
D=[A(\|)] /[A(\perp)]
$$

This is related to the Hermans orientation function of the transition moment direction by the Fraser relationship ${ }^{45}$

$$
\left[\mathrm{P}_{2}\right]_{\mathrm{T}}=(D-1) /(D+2)
$$

A particular IR band is associated with a particular normal mode of vibration of a molecule. In many cases, the normal mode primarily involves localized motions of a particular part of a molecule, so that its transition moment characterizes the orientation of that part. The orientation of a part of a molecule may be associated with a director $a$, a unit vector describing the orientation of that part (for example, a normal to a benzene ring in polystyrene, or a line bisecting the $\mathrm{H}-\mathrm{C}-\mathrm{H}$ bond angle in $\mathrm{PE}$ ).

Another orientation function $\left[\mathrm{P}_{2}\right]_{0}$ is defined by

$$
\left[\mathrm{P}_{2}\right]_{0}=\left[3\left\langle\cos ^{2} \psi\right\rangle-1\right] / 2
$$

where $\psi$ is the angle between $a$ and the transition moment direction, $T$, and defines the average degree of orientation of $a$ with respect to $T$. The orientation of the group of concern is then given by

$$
\left[\mathrm{P}_{2}\right]_{\mathrm{A}}=\left[\mathrm{P}_{2}\right]_{0}\left[\mathrm{P}_{2}\right]_{\mathrm{T}}
$$

Thus, by choosing a suitable IR frequency, one may selectively characterize the orientation of a particular part of a molecule or polymer structure. For example, measurements at $730 \mathrm{~cm}^{-1}$ characterize the orientation of the $a$-axis of PE. In this respect, IRD is superior to birefringence, which measures an average orientation. Some examples of the application of this concept are: (1) the measurement of the separate orientation of the crystalline and amorphous regions of a crystalline polymer; (2) the measurement of the orientation of various polymorphic crystalline forms (as in $\mathrm{PVF}_{2}$ ); (3) the measurement of the orientation of a component of a blend (like $\mathrm{PVF}_{2}$ in $\mathrm{PVF}_{2} / \mathrm{PMMA}$ ); (4) the measurement of the orientation of a component of a copolymer; and (5) the measurement of the orientation of a labelled species in a mixture (PSD of a particular molecular weight in a polydisperse PSH sample).

The interpretation of these measurements is dependent upon obtaining a value of $\left[\mathrm{P}_{2}\right]_{0}$ characterizing the orientation of the transition moment direction in the molecule. This usually requires a normal mode analysis of molecular vibrations; otherwise, it must be obtained by calibrating against another technique. For example, one may calibrate $\left[\mathrm{P}_{3}\right]_{0}$ for a band associated with a crystal if $\left[\mathrm{P}_{2}\right]_{\mathrm{A}}$ is measured using WAXD.

The use of the IRD technique has been limited because of the difficulty of achieving high precision. For samples having low orientation, the values of $A(\|)$ and $A(\perp)$ are not very different, so that an accurate measure of the dichroism is difficult. In the past, with a dispersion spectrometer these were measured separately and successively, so that it was important to have good instrument stability during the course of measurements. To improve precision, methods were proposed involving rotating polarizer $^{83}$ or chopping techniques, ${ }^{84}$ or the use of two. polarizers in the two beams of a double beam spectrometer. ${ }^{85}$

Dynamic IRD measurements (DIRD) were attempted during dispersion spectrometer days. Notable are the efforts of Gotoh ${ }^{85}$ in studying rubber crystallization, the study of relaxation by Onogi and Asada, ${ }^{86,87}$ and the development and 


\section{R. S. STEIN}
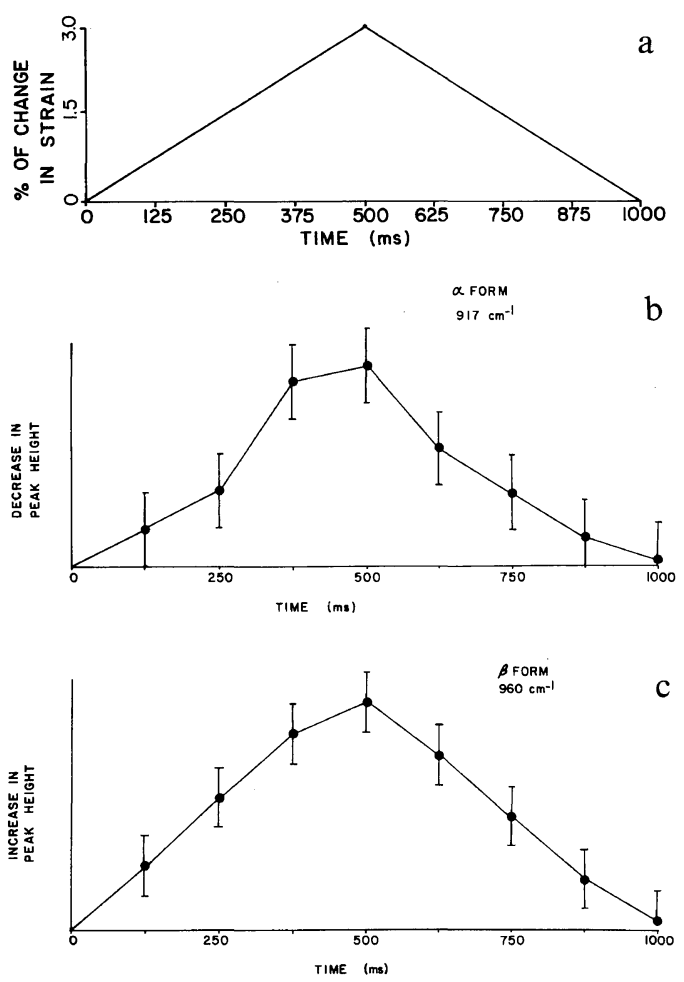

Figure 12. Time resolved FTIR results for poly(tetramethylene terephthalate): (a) applied strain; (b) increase in alpha crystal form; (c) decrease in beta crystal form.

application of a chopper apparatus by Wilkes and Stein. ${ }^{84,88}$ The results were obtained only with the exercize of painstaking effort, and the technique never became popular.

The advent of digital data acquisition and Fourier transform spectroscopy (FTIR) was a breakthrough for the technique. This made possible an improvement in the accuracy of the method through many repetitions of scans. Doing this manually would be too tedious, but with computer control of the spectrometer it becomes feasible. Furthermore, the higher speed of data acquisition with FTIR makes it more reasonable to repeat scans a greater number of times.

These new methods have been applied to the study of time dependent phenomena in the technique of "time-resolved FTIR." ${ }^{89}$ In this method the data scans in obtaining the Fourier transform are synchronized with the time variation of the system being studied, for example, a varying electri- cal or mechanical field applied to the sample. In one-shot experiments where it is desirable to obtain both $\|$ and $\perp$ data in a single experiment, it is possible to rapidly modulate the polarization of the IR beam using piezo-electric techniques. For experiments with periodic fields where one is exploring periodic orientation changes under steady-state conditions, it suffices to change the polarization direction mechanically.

Pioneering work in the application of timeresolved FTIR techniques to polymer problems has been carried out in Amherst by Prof. S. L. Hsu. I will not try to review his accomplishments in this paper but will simply mention one significant application. Figure 12 shows the reversible stressinduced phase transformation in poly(tetramethylene terephthalate $)^{90}$ The sample film was subjected to a $4 \%$ strain amplitude at a frequency of one hertz. The time resolution achieved was $100 \mathrm{~ms}$. The theoretical limit is approximately $20 \mathrm{~ms}$. It shows for the first time that the phase transformation occurs instantly when the stress is applied.

\section{CONCLUSIONS}

Significant progress has been made in developing and improving rheo-optical techniques in the past decade. This has been a consequence to the availability of new probes (neutrons), new sources of high flux radiation (lasers and synchrotrons), new detection techniques (two-dimensional OMA's and position sensitive X-ray detectors), and new data analysis methods (time averaging of periodic signals, FTIR, and time-resolved spectroscopy). In most cases, the instruments are computer interfaced, and their use is feasible because of the availability of fast, economical computers with large storage capacity.

The development of new experimental methods has been accompanied by theoretical advances for their interpretation. In most cases, this involves mathematical modeling of the phenomena of interest. Examples are the improvement of models for spherulite deformation and the Monte Carlo modeling of the chain traverses in crystalline polymers for calculation of $P(q)$ for SANS.

These advances confirm the belief of the author that complex phenomena such as the rheology of crystalline polymers can best be studied by a concerted use of a number of related techniques. Their 
application is symbiotic, in that each new technique often provides information to help understand the results acquired using previous techniques. With rapid advances in electronics, instrumentation and computer technology, we may be optimistic that this will be a continuing and accelerating trend.

Acknowledgments. The author acknowledges with thanks the support of the Materials Research Laboratory of the University of Massachusetts, the Division of Materials Research of the National Science Foundation, and the Army Research Office (Durham). Grants-in-Aid from Monsanto Company, Exxon, and the Millikan Corporation are also appreciated.

Measurements of SANS and were made at the National Center for Small-Angle Scattering Research at Oak Ridge National Laboratory, where the assistance of Dr. G. D. Wignall and Dr. J. S. Lin is particularly appreciated. The loan of a servocontrolled dynamic straining apparatus by the Army Materials and Mechanics Laboratory, Watertown, Massachusetts, is also appreciated.

Much of this paper summarizes contributions from the author's graduate students and associates. With some fear of omission, I might particularly mention R. Tabar, D. Lefebvre, G. Stack and C. Murray (for SALS); S. Suehiro, S. Baczek, T. Kyu and P. Young (for SAXS; R. Hendricks, formerly at ORNL and now at TEC Corporation, was active in the initial stages of design and construction of the DSAXS apparatus); and G. Hadziioannou, R. Lo, and A. Hill (for SANS). Finally, the help of P. Rogers in preparing this manuscript is much appreciated.

\section{REFERENCES}

1. R. S. Stein and S. Onogi, Ed., J. Polym. Sci., C, No. 15 (1966).

2. R. S. Stein, Ed., J. Polym. Sci., C, No. 5 (1964).

3. Symposium in honor of R. S. Stein upon his receiving the American Chemical Society Award in Polymer Chemistry, Seattle, 1983.

4. Polym. Eng. Sci., 24, (1984), special issue devoted to above symposium.

5. R. S. Stein, S. Onogi, and D. A. Keedy, J. Polym. Sci., 57, 801 (1962); R. S. Stein, S. Onogi, K. Sasaguri, and D. A. Keedy, J. Appl. Phys., 34, 80 (1963).

6. R. Yamada and R. S. Stein, J. Appl. Phys., 36, 3005 (1965).
7. T. Hashimoto, R. E. Prud'homme, D. A. Keedy, and R. S. Stein, J. Polym. Sci., Polym. Phys. Ed., 11, 693 (1973)

8. T. Hashimoto, R. E. Prud'homme, and R. S. Stein, J. Polym. Sci., Polym. Phys. Ed., 11, 709 (1973).

9. T. Ito, T. Oda, H. Kawai, T. Kawaguchi, D. A. Keedy, and R. S. Stein, Rev. Sci. Instr., 39, 1847 (1968).

10. A. Tanaka, E. P. Chang, B. Delf, I. Kimura, and R. S. Stein, J. Polym. Sci., Polym. Phys. Ed., 11, 1891 (1973).

11. A. Plaza and R. S. Stein, J. Polym. Sci., 40, 267 (1959).

12. M. B. Rhodes, D. A. Keedy, and R. S. Stein, J. Polym. Sci., 62, S73 (1962).

13. S. Clough, M. B. Rhodes, and R. S. Stein, J. Polym. Sci., C, 18, 1 (1967).

14. P. F. Erhardt and R. S. Stein, Appl. Polym. Symposia, 5, 113.(1967).

15. R. S. Stein and M. B. Rhodes, J. Appl. Phys., 31, 1873 (1960).

16. R. S. Stein and A. Misra, J. Polym. Sci., Polym. Phys. Ed., 11, 109 (1973).

17. R. S. Stein and W. Chu, J. Polym. Sci., A-2, 8, 1137 (1970).

18. D. Y. Yoon and R. S. Stein, J. Polym. Sci., Polym. Phys. Ed., 12, 763 (1974).

19. D. Y. Yoon and R. S. Stein, J. Polym. Sci., Polym. Phys. Ed., 12, 735 (1974).

20. A. Wasiak, D. Peiffer, and R. S. Stein, J. Polym. Sci., Polym. Lett. Ed., 14, 381 (1976).

21. R. J. Tabar, R. S. Stein, and M. B. Long, J. Polym. Sci., Polym. Phys. Ed., 20, 2041 (1982).

22. R. J. Samuels, J. Polym. Sci., A-2, 8, 1101 (1968).

23. R. J. Samuels, "Structured Polymer Properties," Interscience Publishers, New York, 1974.

24. S. Clough, J. J. van Aartsen, and R. S. Stein, J. Appl. Phys., 36, 3072 (1965).

25. J. J. van Aartsen and R. S. Stein, J. Polym. Sci., A-2, 9, 295 (1971).

26. A. Todo, T. Hashimoto, and H. Kawai, Polym. J., 11, 59 (1970).

27. M. Matsuo, J. Chem. Phys., 72, 899 (1980).

28. P. H. Geil, "Polymer Single Crystals," Interscience Publishers, New York, 1963.

29. T. T. Wang, J. Polym. Sci., Polym. Phys. Ed., 12, 145 (1974).

30. D. Lefebvre, R. J. Tabar, and R. S. Stein, in preparation.

31. V. Petraccone, I. C. Sanchez, and R. S. Stein, J. Polym. Sci., Polym. Phys. Ed., 13, 1991 (1975).

32. M. B. Rhodes and R. S. Stein, J. Polym. Sci., A-2, 7, 1539 (1969).

33. N. Hayushi and H. Kawai, Polym. J., 3, 140 (1972).

34. A. Guinier and G. Fournet, "Small Angle Scattering of X-rays," John Wiley and Sons, New York, 1955.

35. C. Vonk, J. Appl. Cryst., 6, 81 (1973). 
36. R. Hoseman and S. N. Bagchi, "Direct Analysis of Diffraction by Matter," North-Holland Publishing Co., Amsterdam, 1962.

37. I. L. Hay and A. Keller, J. Mater. Sci., 1, 41 (1965); ibid., 2, 538 (1966).

38. S. Baczek, "Small Angle X-ray Studies of the Deformation of Polyethylene," $\mathrm{Ph}$. D. dissertation, University of Massachusetts, Amherst, 1977.

39. C. J. Borowski and M. K. Kopp, Rev. Sci. Instr., 39, 1515 (1968).

40. T. P. Russell, R. S. Stein, M. K. Kopp, R. E. Zedler, R. W. Hendricks, and J. S. Lin, Oak Ridge National Laboratory Report, ORNL-6678, 1979.

41. R. W. Hendricks, J. Appl. Cryst., 11, 15 (1978).

42. J. S. Higgins and R. S. Stein, J. Appl. Cryst., 11, 346 (1978).

43. G. D. Wignall, R. W. Hendricks, W. C. Koehler, J. S. Lin, M. P. Wai, E. L. Thomas, and R. S. Stein, Polymer, 22, 886 (1981).

44. H. Benoit, D. Decker, R. Duplessix, C. Picot, P. Rempp, J. P. Cotton, B. Farnoux, G. Jannink, and R. Ober, J. Polym. Sci., Polym. Phys. Ed., 14, 2119 (1976).

45. R. D. B. Fraser, J. Chem. Phys., 21, 1511 (1953).

46. R. S. Stein, J. Polym. Sci., 31, 327 (1958).

47. R. S. Stein and F. H. Norris, J. Polym. Sci., 21, 381 (1956).

48. B. E. Read and R. S. Stein, Macromolecules, 1, 116 (1968).

49. R. J. Cembrola, T. Kyu, and R. S. Stein, J. Polym. Sci., Polym. Phys. Ed., 21, 329 (1983).

50. S. Suehiro, T. Yamada, H. Inagaki, and H. Kawai, Polym. J., 10, 315 (1978).

51. R. J. Tabar, "Quantitative Small-Angle Light Scattering Studies of Semicrystalline Polymers, Ph. D. dissertation, University of Massachusetts, Amherst, 1983.

52. R. J. Tabar, P. Leite-James, and R. S. Stein, submitted for publication.

53. R. J. Tabar, D. Lefebvre, and R. S. Stein, in preparation.

54. R. J. Tabar, A. Wasiak, S. D. Hong, T. Yuasa, and R. S. Stein, J. Polym. Sci., Polym. Phys. Ed., 19, 49 (1981).

55. R. J. Tabar, R. S. Stein and D. E. Rose, submitted for publication.

56. S. Suehiro, R. W. Hendricks, J. S. Lin, T. Kyu, P. Young, and R. S. Stein, in preparation.

57. P. Doty, B. H. Zimm and H. Mark, J. Phys. Chem., 12, 144 (1944); ibid., 13, 159 (1945).

58. G. Hadziioannou, L. Wang, R. S. Stein, and R. S. Porter, Macromolecules, 15, 880 (1982).

59. P. D. Griswold, A. E. Zachariades, and R. S. Porter, in "Flow-Induced Crystallization in Polymer Systems," R. L. Miller, Ed., Gordon and Breach, New York, 1979, pp 205-211.

60. A. Lodge, "Elastic Liquids," Academic Press, New
York, 1964.

61. P. G. deGennes, J. Chem. Phys., 55, 572 (1971).

62. M. Doi and S. F. Edwards, J. Chem. Soc., Faraday Trans. 2, 74, 1789 (1978).

63. W. W. Graessley, Faraday Symp. Chem. Soc., 18, 1 (1983).

64. G.. Hadziioannou and R. Lo, unpublished work, 1983.

65. F. Boue, M. Nierlich, G. Jannink, and R. Ball, J. Physique, 43, 137 (1982).

66. R. S. Stein, S. Onogi, K. Sasaguri, and D. A. Keedy, J. Appl. Phys., 34, 80 (1963).

67. D. C. Anderson, "Birefringence Relaxation Studies of Polyethylene," Ph. D. dissertation, University of Massachusetts, Amherst, 1981.

68. S. Onogi, T. Asada, Y. Fukui, and T. Fujisawa, $J$. Polym. Sci., A-2, 5, 1067 (1967).

69. T. Kyu, N. Yasuda, M. Tabushi, S. Nomura, and H. Kawai, Polym. J., 7, 108 (1975).

70. P. H. Hermans, "Contributions to the Physics of Cellulose Fibers," Elsevier, New York, 1946, p 198.

71. W. Kuhn and F. Grun, Kolloid Z., Z. Polym., 101, 248 (1942).

72. P. Debye, Technical Report No. 637 to Rubber Reserve Company, April 9, 1945.

73. P. J. Flory, "The Statistical Mechanics of Chain Molecules," Interscience Publishers, New York, 1968.

74. D. Y. Yoon and P. J. Flory, Polym. Bul., 4, 693 (1981).

75. J. Schelten, G. D. Wignall, D. G. H. Ballard, and G. W. Longman, Polymer, 18, 1111 (1977).

76. G. D. Wignall and W. Wu, Polym. Commun., 24, 354 (1983).

77. W. Graessley and B. Crist, private communication, 1984.

78. D. G. H. Ballard, A. N. Burgess, A. Nevin, P. Cheshire, G. W. Longman, and J. Schelten, Macromolecules, 13, 677 (1980).

79. D. Y. Yoon, J. Appl. Cryst., 11, 531 (1978).

80. J. Schelten, D. G. H. Ballard, G. D. Wignall, G. Longman, and W. Schmatz, Polymer, 17, 751 (1976).

81. A. Hill, R. Lo, R. S. Porter, and R. S. Stein, in preparation.

82. E. W. Fischer, K. Hahn, J. Kugler, U. Struth, R. Born, and M. Stamm, J. Polym. Sci., Polym. Phys. Ed., 22, 1491 (1984).

83. R. S. Stein, J. Appl. Polym. Sci., 5, 96 (1961).

84. G. L. Wilkes, "Rheo-Optical Studies of Some Copolymers, Ph. D. dissertation, University of Massachusetts, Amherst, 1969.

85. R. Gotoh, T. Takenaka, and N. Hayama, Kolloid Z., Z. Polym., 205, 18 (1965).

86. S. Onogi and T. Asada, J. Polym. Sci., C, 16, 1445 (1967).

87. S. Onogi, T. Asada, and K. Sakai, "Proceedings of the Fifth International Congress on Rheology," 


\section{Progress in Rheo-Optics}

S. Onogi, Ed., University of Tokyo Press, Tokyo, 89. J. E. Lasch, D. J. Burchell, T. Masaoka, and S. L. 1970 , pp $41-50$. Hsu, Appl. Spectroscopy, 38, 351 (1984).

88. Y. Uemura, R. S. Stein, and W. J. MacKnight, 90. S. L. Hsu, private communication, 1984. Macromolecules, 4, 490 (1971). 